\title{
Homossexualidade e bissexualidade feminina no SUS: Ações em saúde realizadas pela atenção básica
}

Homosexuality and female bisexuality in the SUS: Health actions carried out by primary care Homosexualidad y bisexualidad femenina en el SUS: Acciones de salud llevadas a cabo por atención primaria

Stéffane Costa Mendes ORCID: https://orcid.org/0000-0002-9004-9515 Universidade Regional do Cariri, Brasil E-mail: Steffaneecostam@Gmail.Com

Antonio Wellington Vieira Mendes ORCID: https://orcid.org/0000-0001-8526-6964 Universidade Regional do Cariri, Brasil

E-mail: Wellingtonmendes723@Gmail.Com Andreza Vitor da Silva ORCID: https://orcid.org/0000-0002-9999-4986 Universidade Regional do Cariri, Brasil E-mail: Andrezavitor.Sv@Gmail.Com

Carolaine da Silva Souza ORCID: https://orcid.org/0000-0002-6369-5749 Universidade Regional do Cariri, Brasil E-mail: Carolainec856@Gmail.Com

Darlyanne Chaves Feitosa Araujo ORCID: https://orcid.org/0000-0003-4307-2090 Secretaria Municipal de Saúde de Iguatu, Brasil E-mail: Darlyanneenfa@Gmail.Com

João Paulo Xavier Silva

ORCID: http://orcid.org/0000-0003-3082-9373 Universidade Regional do Cariri, Brasil

E-mail: Jpxavier.Enf@Gmail.Com José Gerfeson Alves

ORCID: https://orcid.org/0000-0003-0364-3151 Universidade Regional do Cariri, Brasil

E-mail: Gerfesondip@Gmail.Com

Karina Ellen Alves de Albuquerque

ORCID: https://orcid.org/0000-0002-5317-5600 Universidade Regional do Cariri, Brasil E-mail: Karinaellenalves2@Gmail.Com Lorena Pinheiro Braga

ORCID: https://orcid.org/0000-0001-8251-1784 Universidade Regional do Cariri, Brasil E-mail: Lorenabraga631@Gmail.Com

Maria Socorro da Silva Primo

ORCID: https://orcid.org/0000-0001-6387-8775 Secretaria Municipal de Saúde de Varzea Alegre, Brasil

E-mail: Socorro_Help_2009@Hotmail.Com

Mariana Cordeiro da Silva

ORCID: https://orcid.org/0000-0002-2934-0338 Universidade Regional do Cariri, Brasil

E-mail: Mariana.Cordeiro110@Gmail.Com

Marina da Silva dos Santos

ORCID: https://orcid.org/0000-0002-7234-8933 Universidade Regional do Cariri, Brasil E-mail: Marina.Silva@Urca.Br

Samara Calixto Gomes

ORCID: https://orcid.org/0000-0002-7929-3648 Universidade Regional do Cariri, Brasil E-mail: Samaracalixto@Hotmail.Com 


\title{
Resumo
}

O Sistema Único de Saúde viabiliza a promoção da universalidade, assim, em 2011 foi instituída a Política Nacional de Saúde Integral de Lésbicas, Gays, Bissexuais, Travestis e Transexuais. Mulheres lésbicas e bissexuais enfrentam inúmeros obstáculos na busca por acesso aos serviços de saúde, podendo estar relacionado a invisibilidade da sexualidade feminina, até o preconceito por sua orientação sexual. Nota-se que o profissional de enfermagem é o que está mais próximo da população nos serviços de saúde, especialmente na Atenção Básica. O estudo objetivou identificar a assistência de saúde para mulheres lésbicas e bissexuais realizada na atenção básica. Trata-se de uma Revisão Integrativa (RI) sendo desenvolvida seguindo os seis passos proposto por Mendes, Silveira e Galvão (2019). A busca e seleção de artigos foi realizada no período de Agosto e Setembro de 2020, nas bases de dados e portais: MEDLINE IBECS, BDENF, via portal Biblioteca Virtual em saúde (BVS), LILACS e SCIELO pelo portal CAPES. Utilizando os descritores "Homossexualidade Feminina", "Bissexualidade" e "Integralidade em Saúde", por meio do operador booleano "AND”. Mulheres lésbicas e bissexuais não são acolhidas e assistidas de maneira integral, de acordo com suas necessidades de saúde. A falta de informação, preconceito ou desinteresse em relação a inclusão e especificidades dessas mulheres avalia-se como preocupante. Percebe-se que não é oferecido um ambiente acolhedor para que haja conforto em expressar sua sexualidade, bem como, sanar suas dúvidas.

Palavras-chave: Homossexualidade Feminina; Bissexualidade; Integralidade em saúde.

\begin{abstract}
The Unified Health System enables the promotion of universality, so in 2011 the National Policy for Integral Health of Lesbians, Gays, Bisexuals, Transvestites and Transsexuals was instituted. Lesbian and bisexual women face numerous obstacles in the search for access to health services, which may be related to the invisibility of female sexuality, to prejudice due to their sexual orientation. It is noted that the nursing professional is the one that is closest to the population in health services, especially in Primary Care. The study aimed to identify health care for lesbian and bisexual women performed in primary care. This is an Integrative Review (IR) being developed following the six steps proposed by Mendes, Silveira and Galvão (2019). The search and selection of articles was carried out in August and September 2020, in the databases and portals: MEDLINE, IBECS, BDENF, via the Virtual Health Library (VHL), LILACS and SCIELO portal through capes portal. Using the descriptors "Female Homosexuality", "Bisexuality" and "Integrality in Health", through the Boolean operator "AND". Lesbian and bisexual women are not fully welcomed and assisted according to their health needs. The lack of information, prejudice or lack of interest in relation to the inclusion and specificities of these women is evaluated as worrying. It is perceived that there is no welcoming environment for there to be comfort in expressing your sexuality, as well as, to answer their doubts.
\end{abstract}

Keywords: Female homosexuality; Bisexuality; Integrality in health.

\section{Resumen}

El Sistema Unificado de Salud permite la promoción de la universalidad, por lo que en 2011 se instituyó la Política Nacional de Salud Integral de Lesbianas, Gays, Bisexuales, Travestis y Transexuales. Las mujeres lesbianas y bisexuales se enfrentan a numerosos obstáculos en la búsqueda del acceso a los servicios de salud, que pueden estar relacionados con la invisibilidad de la sexualidad femenina, a los prejuicios debido a su orientación sexual. Cabe destacar que el profesional de enfermería es el más cercano a la población en los servicios sanitarios, especialmente en Atención Primaria. El estudio tenía como objetivo identificar la atención médica para mujeres lesbianas y bisexuales realizadas en atención primaria. Se trata de una revisión integrativa (IR) que se está desarrollando, siguiendo los seis pasos propuestos por Mendes, Silveira y Galvão (2019). La búsqueda y selección de artículos se llevó a cabo en agosto y septiembre de 2020, en las bases de datos y portales: MEDLINE, IBECS, BDENF, a través de la Biblioteca Virtual de Salud (VHL), LILACS y el portal SCIELO a través del portal capes. Utilizando los descriptores "Homosexualidad Femenina", "Bisexualidad" e "Integralidad en Salud", a través del operador booleano "AND". Las mujeres lesbianas y bisexuales no son plenamente bienvenidas y asistidas de acuerdo con sus necesidades de salud. La falta de información, prejuicios o falta de interés en relación con la inclusión y especificidad de estas mujeres se considera preocupante. Se percibe que no hay un ambiente acogedor para que haya consuelo en expresar su sexualidad, así como, para responder a sus dudas.

Palabras clave: Homosexualidad femenina; Bisexualidad; Integralidad en salud. 


\section{Introdução}

A implantação do SUS (Sistema Único de Saúde) viabiliza a promoção da universalidade no acesso, maior equidade e integralidade do cuidado. A Atenção Básica, consolidada por meio da Estratégia Saúde da Família (ESF) como porta de entrada dos serviços de saúde, é responsável por garantir o acesso da população à assistência de qualidade com cuidado integral, sensível às suas especificidades, humanizado, livre de discriminação étnica, sociocultural, religiosa e sexual (Alves, Gonçalves. 2016; Oliveira, et al. 2018).

Afim de promover a inclusão, reduzir barreiras socioculturais e desigualdades de acesso de grupos, em dezembro de 2011 foi instituída a Política Nacional de Saúde Integral de Lésbicas, Gays, Bissexuais, Travestis e Transexuais (PNSILGBT), reconhecendo efeitos da exclusão e discriminação no processo saúde-doença destes grupos. Contém estratégias, metas e objetiva reduzir preconceito institucional, garantindo assistência de qualidade e resolutiva às demandas específicas de saúde, introdução gradual de documentos sobre a orientação sexual, reconhecimento de nome social e identidade de gênero (Brasil, 2013; Oliveira, et al. 2018; Silva; Prata, 2018).

Contudo, mulheres lésbicas e bissexuais enfrentam inúmeros obstáculos na busca por acesso aos serviços de saúde, podendo estar relacionado a invisibilidade da sexualidade feminina, até o preconceito por sua orientação sexual. Falar sobre saúde física e mental dessas mulheres é pontuar sobre apagamentos e violências, pois suas vivências são construídas com uma carência de referências que tornem válido o seu lugar social e familiar, tal como sua forma de se relacionar afetiva e sexualmente, isso já se torna fator de adoecimento (Oliveira, et al. 2018; Fernandes; Soler; Leite, 2018).

Suas especificidades na saúde muitas vezes ainda não são observadas e inclinam-se a procurar os serviços de atendimento com menor frequência, sendo citadas como causas, experiências de discriminação e condutas inadequadas no atendimento pelos profissionais de saúde, resultado do preconceito, desinformação sobre as demandas de saúde dessa população e a negação pelas próprias mulheres do risco para determinadas doenças (Araújo, et al. 2019; Crispim, et al. 2018).

A maior parte dos cuidados, assistência e orientação em saúde sexual e reprodutiva é realizado na atenção básica pela equipe de saúde, em especial, pelo enfermeiro. Portanto, é reconhecido o papel essencial e participativo da equipe multidisciplinar de profissionais na atenção e assistência prestados à esse grupo

Diante das razões apontadas, o estudo fez-se necessário por conta das demandas de saúde dessa população, reconhecendo sua situação de vulnerabilidade, consequências do preconceito e discriminação no processo saúde-doença e necessidade de visibilidade e atendimento adequado às suas especificidades de saúde que são estendidas ao profissional enfermeiro que atua na ESF.

Objetivou-se identificar por meio da literatura a assistência de saúde para mulheres lésbicas e bissexuais realizada pelo enfermeiro da atenção básica, afim de contribuir para o meio acadêmico, da saúde pública e sociedade, despertando a curiosidade e criticidade sobre a temática e promover maior visibilidade para produção científica nesta área.

\section{Metodologia}

Trata-se de uma Revisão Integrativa (RI), onde consiste em reunir e sintetizar conhecimento científico sendo desenvolvido seguindo os seis passos proposto por Mendes, Silveira e Galvão (2019). 1: Definição do tema, objetivo e pergunta de revisão para o desenvolvimento do estudo. 2: Estabelecimento de critérios de inclusão e exclusão, base de dados, busca e seleção de estudos. 3: Extração e organização de dados. 4: Avaliação dos estudos incluídos na revisão. 5: Síntese dos resultados da revisão. 6: Elaboração de documento para apresentação da revisão.

Buscou-se responder à questão norteadora: Quais as ações em saúde realizadas na atenção básica para o cuidado integral de mulheres lésbicas e bissexuais evidenciadas na literatura? 
Para escolha e elaboração dos descritores de busca foi utilizada a estratégia Population, Variable and Outcomes $(P V O)$, que proporciona organizar os elementos da pesquisa da seguinte forma, Population: Definir população, contexto e/ou situação, Variable: Variáveis do estudo e Outcomes: desfecho ou resultados desejados.

Quadro 1: Estratégia para definição de Descritores através da PVO, Iguatu/CE, 2020.

\begin{tabular}{|c|c|c|}
\hline Itens da Estratégia & Componentes & Descritores de Assunto \\
\hline Population & Mulheres lésbicas e bissexuais & $\begin{array}{c}\text { Homossexualidade feminina } \\
\text { Bissexualidade }\end{array}$ \\
\hline Variable & Cuidados de saúde & Cuidados de saúde \\
\hline Outcomes & Integralidade em Saúde & Integralidade em Saúde \\
\hline
\end{tabular}

Fonte: Autores.

A busca e seleção de artigos foi realizada no período de Agosto e Setembro 2020, nas bases de dados e portais: Sistema Online de Busca e Análise de Literatura Médica (MEDLINE), Base de dados em Enfermagem (BDENF), via portal Biblioteca Virtual em saúde (BVS), Centro Latino- Americano e do Caribe de Informações em Ciências da Saúde (LILACS), Scientific Electronic Library Online (SCIELO), pelo portal de periódicos da Coordenação de Aperfeiçoamento de Pessoal de Nível Superior (CAPES) e Índice Bibliográfico Espanhol de Ciências de Saúde (IBECS). Foram utilizados os descritores, “Homossexualidade Feminina", "Bissexualidade" e "Integralidade em Saúde”, por meio do operador booleano "AND”.

Adotamos como critérios de inclusão serem artigos completos, em formato original, publicados de 2013 a 2020 , nos idiomas português, inglês e espanhol. Já os critérios de exclusão foram as publicações que não respondessem a pergunta de pesquisa, artigos repetidos e duplicados. Ressalta-se que o estabelecimento deste período de tempo se deu por conta do marco histórico onde a Política Nacional de Saúde Integral de Lésbicas, Gays, Bissexuais, Travestis e Transexuais foi implementada no ano de 2013 pelo Ministério da Saúde.

Após a identificação, os artigos foram submetidos a um processo de triagem, análise do assunto, por meio da leitura do título e resumo. Depois foi realizado a leitura do texto completo dos artigos selecionados pelos critérios de inclusão e exclusão. Os artigos duplicados entre bases de dados e repetidos entre os selecionados foram identificados, tendo sido realizada a seleção definitiva das referências elegíveis. Utilizou-se o instrumento Preferred Reporting Items for Systematic Review and Meta-Analyses (PRISMA) para relatar o processo de identificação, triagem, elegibilidade e inclusão dos estudos, conforme representado na Figura 1. 
Figura 1 - Fluxograma de seleção de estudos, Iguatu/CE, 2020.

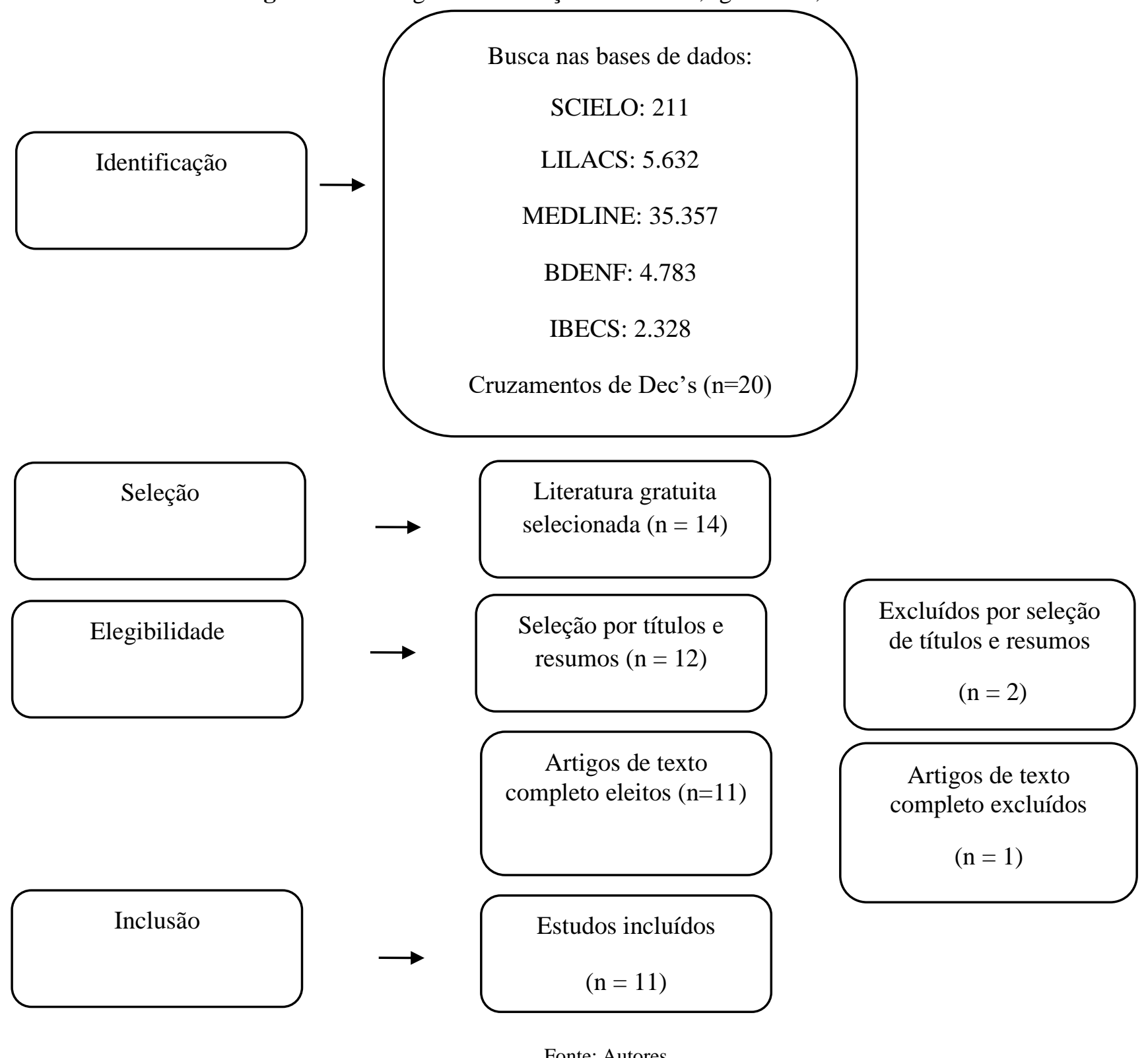

\section{Resultados}

A amostra final do estudo conta com um total de 11 artigos referentes à temática, e foram apresentados segundo base de dados, título, ano e tipo de estudo. 
Quadro 3: Caracterização dos estudos incluídos, segundo base de dados, título, ano e tipo de estudo, Iguatu/CE, 2020.

\begin{tabular}{|c|c|c|}
\hline Base de dados & Título/Ano & Tipo de estudo \\
\hline MEDLINE & $\begin{array}{c}\text { Mental Health and Substance Use Disparities } \\
\text { Among Urban Adolescent Lesbian and Bisexual } \\
\text { Girls, } 2013 .\end{array}$ & $\begin{array}{l}\text { Estudo prospectivo de coorte } \\
\text { múltipla. }\end{array}$ \\
\hline MEDLINE & $\begin{array}{c}\text { The Relationships of Sexual Identity, } \\
\text { Hazardous Drinking, and Drinking } \\
\text { Expectancies with Risky Sexual Behaviors in a } \\
\text { Community Sample of Lesbian and Bisexual } \\
\text { Women, } 2013 \text {. }\end{array}$ & Estudo Longitudinal. \\
\hline MEDLINE & Open the door for LGBTQ patients, 2013. & Estudo descritivo qualitativo \\
\hline MEDLINE & $\begin{array}{c}\text { Magnet Nurse Administrator Attitudes and } \\
\text { pportunities, } 2014 .\end{array}$ & Estudo descritivo qualitativo. \\
\hline MEDLINE & $\begin{array}{l}\text { Improving Nursing Care for Lesbian, Bisexual, } \\
\text { and Transgender Women, } 2014\end{array}$ & Estudo de coorte. \\
\hline MEDLINE & $\begin{array}{c}\text { Survey of lesbian, gay, bisexual, and } \\
\text { transgender people's experiences of mental } \\
\text { health services in Ireland, } 2014\end{array}$ & Estudo exploratório. \\
\hline MEDLINE & $\begin{array}{l}\text { Stakeholders' Recommendations to Improve } \\
\text { Patient-centered “LGBTQ” Primary Care in } \\
\text { Rural and Multicultural Practices, } 2016 .\end{array}$ & Estudo descritivo, qualitativo \\
\hline MEDLINE & $\begin{array}{l}\text { Caring for lgbtq youth in inclusive and } \\
\text { affirmative environments, } 2016 .\end{array}$ & Estudo Longitudinal \\
\hline MEDLINE & $\begin{array}{l}\text { Do student nurses feel a lack of comfort in } \\
\text { providing support for Lesbian, Gay, Bisexual or } \\
\text { Questioning adolescents: what factors } \\
\text { inflfluence their comfort level?, } 2017 .\end{array}$ & $\begin{array}{l}\text { Estudo quantitativo e } \\
\text { qualitativo. }\end{array}$ \\
\hline MEDLINE & $\begin{array}{c}\text { Creating LGBTQ-Inclusive Care and Work } \\
\text { Environments, } 2018 .\end{array}$ & Estudo exploratório \\
\hline BDENF & $\begin{array}{l}\text { Assistência de enfermagem às mulheres } \\
\text { lésbicas e bissexuais, } 2019 .\end{array}$ & $\begin{array}{l}\text { Estudo qualitativo, } \\
\text { exploratório e descritivo }\end{array}$ \\
\hline
\end{tabular}

Fonte: Autores.

Os estudos foram publicados entre 2013 e 2019, sendo grande parte entre os anos de 2013 e 2016, dez são de idioma inglês e um português, dos dez em inglês oito tem como país de origem os Estados Unidos (Marshal, et al. 2013; Mattews, et al. 2013; Eliason, et al. 2013; Klotzbaugh \& Spancer, 2014; Zuzelo, 2014; Kano, et al. 2016; Hadland; Yehia \& Makadon., 2016; Jones-Schenk \& Bleich, 2018), um na Irlanda (Mccann \& Sharek, 2014), um no Reino Unido (Richardso; Ondracek \& Anderson, 2017) e um no Brasil (Cabral et al. 2019). 
Relacionado aos aspectos metodológicos dez são de abordagem qualitativa (Marsha,1 et al. 2013; Mattews, et al. 2013; Eliason, et al, 2013; Klotzbaugh \& Spancer, 2014; Zuzelo, 2014; Kano, et al, 2016; Hadland; Yehia \& Makadon., 2016; Jones-Schenk \& Bleich, 2018; Mccann \& Sharek, 2014; Cabral et al., 2019) e um com abordagem mista, qualitativa e quantitativa (Richardso; Ondracek \& Anderson, 2017).

As metodologias aplicadas foram quatro por meio de entrevista semiestruturada (Kano et al., 2016; Hadland et al., 2016; Jones-Schenk \& Bleich, 2018; Cabral et al., 2019) e sete houve aplicação de questionário (Marshal et al., 2013; Mattews, et al., 2013; Eliason et al., 2013; Zuzelo, 2014; Mccann \& Sharek, 2014; (Richardso; Ondrace \& Anderson, 2017); Klotzbaugh \& Spancer, 2014), onde um deles foi por envio eletrônico.

No que se refere as populações dos estudos três incluíram mulheres lésbicas e bissexuais (MARSHAL et al., 2013; Mattews et al., 2013; Cabral et al., 2019), um estudantes do curso de enfermagem ( Richardso; Ondracek \& Anderson, 2017), cinco incluíram profissionais de enfermagem (Eliason et al., 2013; Klotzbaugh \& Spancer, 2014; Zuzelo, 2014; Hadland; Yehia \& Makadon., 2016; Jones-schenk \& Bleich, 2018) e dois incluíram pessoas da população LGBT (Mccann \& Sharek, 2014; Kano et al., 2016).

\section{Discussão}

Os estudos incluídos nessa revisão nos mostraram dificuldades no acesso da população LGBTQ aos serviços de saúde e muitas vezes negligência das suas necessidades em saúde causados principalmente pelo desconhecimento dos profissionais do serviço da política LGBTQ, não abordagem desse tema na formação acadêmica e falha no processo de educação permanente dos profissionais.

Muitos estudos falam sobre a vulnerabilidade de pessoas LGBTQ em sofrer violência, discriminação, negligência e assédio, quando os profissionais de saúde não têm conhecimento sobre suas especificidades. Assim, é necessário expandir na área da saúde abordagens mais amplas de questões raciais, étnicas, diversidade sexual, idade, classe social e incapacidades (Eliason et al., 2013).

Mulheres lésbicas, muitas vezes, passam despercebidas nos serviços de saúde, pois a identificação da sua orientação sexual é realizada pela identificação de características geralmente atribuídas ao sexo masculino, quando tais traços não são percebidos, as mesmas são atendidas como heterossexuais, assim, a assistência e orientações não são adequadas às suas necessidades e suas demandas específicas não são identificadas (Silva \& Prata, 2018).

As dificuldades de acesso ao serviço de saúde enfrentadas pela população LGBTQ foram identificadas em vários estudos, e isso pode contribuir para essa população evitar procurar assistência devido ao medo de sofrer discriminação, e mesmo uma vez sendo atendido, há a possibilidade de ocultar sua orientação sexual durante o atendimento ou temer a violação da sua privacidade (Hadland; Yehia \& Makadon, 2016; Jones-Schenk \& Bbleich, 2018; Mccann \& Sharek, 2014; Kano et al., 2016; Cabral, et al., 2019).

Ainda há ignorância em relação as práticas sexuais entre mulheres, resultando em uma invisibilidade, pois muitas vezes a sexualidade feminina é associada exclusivamente a um homem, de forma passiva, para procriação. Essa invisibilidade, ausência de políticas efetivas e desinformação sobre este grupo interferem no atendimento e atuação dos profissionais de saúde que agem a partir de estereótipos socialmente pré-estabelecidos (Moscheta; Fébole \& Anzolin, 2016).

De acordo com o protocolo de Atenção Básica: Saúde das Mulheres, mulheres lésbicas e bissexuais, possuem direito ao planejamento da vida sexual e reprodutiva, aborto legalizado, tecnologias de reprodução, assistência humanizada nos períodos de gravidez, parto e puerpério, respeitando as diversidades e garantindo acesso aos serviços (Brasil, 2016). 
Nota-se que as discussões sobre a saúde de mulheres lésbicas e bissexuais são ínfimas, contribuindo para que haja maior vulnerabilidade à doenças que normalmente são detectadas em exames preventivos de rotina. Os cuidados e orientações devem ser culturalmente apropriados aos seus valores e vivências, justificando a necessidade de habilidades específicas no atendimento (Crispim et al., 2018; Farias et al., 2018; Sousa et al., 2014).

Percebe-se que serviços essenciais realizados na $A B$ não incluem as mulheres lésbicas e bissexuais, como os exames preventivos para o câncer de mama e de colo de útero, pela ideia equivocada de não haver risco para algumas infecções sexualmente transmissíveis como o Human Papiloma Vírus (HPV). O exame colpocitológico deve ser realizado em todas as mulheres independente de sua orientação sexual, adequando as práticas sexuais vivenciadas pelas mesmas (Kano et al., 2016).

O câncer de ovário tem maior probabilidade de se desenvolver em mulheres que nunca fizeram uso de anticoncepcionais, e o câncer de mama, por conta da nuliparidade, mulheres idosas deste grupo enfrentam barreiras para a saúde por conta de fatores sociais históricos, isolamento, ausência de apoio no círculo familiar, representatividade e falhas em serviços sociais e de saúde culturalmente negligenciando a sexualidade da pessoa idosa (Kano et al., 2016; Jones-Schenk \& Bleich, 2018).

Deste modo, a concepção de que mulheres lésbicas e bissexuais não têm necessidades de orientações sobre a prática do sexo seguro resulta em um equívoco, levando a problemas de saúde pública. Diante disso, a rotina dos atendimentos devese atentar para o reconhecimento da orientação sexual e identidade de gênero do paciente, incluindo além do campo de sexo biológico, na anamnese, prontuários clínicos, ficha de notificação de violência e demais documentos de identificação e notificação (Brasil, 2013).

Identificamos nos estudos que na Rede de Atenção Básica o profissional de saúde que está mais próximo da população, é o enfermeiro, que independentemente do tipo de consulta a ser prestada, deve ser realizada a anamnese. No entanto, nesse processo muitas vezes não tratam de questões sobre a sexualidade das mulheres, embora seja uma necessidade, para assim o profissional direcionar orientações adequadas à demanda, minimizando um ambiente que visualize a mulher apenas enquanto heterossexual, mãe e adulta (Cabral et al., 2019).

Outro ponto foi a identificação da deficiência na formação acadêmica sobre políticas direcionadas à população (LGBTQ) desses profissionais, onde muitas vezes não estão familiarizados com termos usados para se referir a indivíduos desta população. Bem como, as necessidades de cuidados de saúde de mulheres lésbicas e bissexuais não são exploradas de maneira adequada durante o curso de enfermagem (Eliason et al., 2013; Klotzbaugh; Spancer, 2014; Zuzelo, 2014; Mccann \& Sharek, 2014; Hadland;Yehia \& Makadon, 2016; Richardso; Ondracek \& Anderson, 2017).

O profissional enfermeiro assume papel de liderança, reconhecendo a necessidade do compromisso com a inclusão LGBTQ, por meio da verificação de procedimentos e políticas inclusivas. Apesar do acesso a recursos e informações referentes ao atendimento a essa população, um fator importante é a necessidade de sensibilização do profissional, pois o desconhecimento, preconceito e estereótipos contribuem significativamente na relação enfermeiro-paciente e na qualidade do cuidado prestado (Eliason et al., 2013; Zuzelo, 2014; Klotzbaugh \& Spancer, 2014; Richardso; Ondracek \& Anderson, 2017; Cabral et al., 2019).

A fragmentação do cuidado à saúde de mulheres lésbicas e bissexuais, torna urgente e indispensável a sensibilização e capacitação dos profissionais de saúde direcionado as especificidades da população LGBTQ. Faz-se necessário que os enfermeiros e demais profissionais da $\mathrm{AB}$ avaliem sua atuação profissional no que diz respeito à assistência a esse grupo, uma vez que o cuidado integral a saúde deve ser desenvolvido em todos os âmbitos da assistência, principalmente na Atenção Básica.

Nesta perspectiva, é de suma importância que os profissionais tenham maior familiaridade com as demandas apresentadas por estas mulheres, proporcionando visibilidade e olhar crítico as políticas públicas, para o enfrentamento das 
demandas e satisfação das necessidades de saúde desta população. Essa revisão apresentou como limitação a baixa produção científica abordando a temática assistência à população de mulheres lésbicas e bissexuais na Atenção Primária, tornando relevante sua produção que contribuirá como pesquisa científica à acadêmicos, profissionais da área, saúde pública e sociedade.

\section{Conclusão}

Diante do exposto, demonstrou-se que mulheres lésbicas e bissexuais não são acolhidas e assistidas de maneira integral, de acordo com suas necessidades de saúde. A falta de informação, preconceito ou desinteresse em relação a inclusão e especificidades dessas mulheres avalia-se como preocupante. Percebe-se que não é oferecido um ambiente acolhedor para que haja conforto em expressar sua sexualidade, bem como, sanar suas dúvidas.

Verifica-se a partir dos resultados do estudo a importância da participação dos gestores municipais acerca da saúde deste grupo de mulheres, principalmente com a utilização da educação permanente. Há necessidade de criar espaços para trabalhar esta temática no ambiente de trabalho e na grade curricular dos cursos de graduação, buscando a desconstrução de preconceitos e visibilidade dessas mulheres, bem como, a promoção de mais estudos científicos buscando identificar dificuldades no âmbito da saúde, afim de melhorar a assistência de enfermagem.

\section{Referências}

Alves, C. M. R \& Gonçalves, M. A. M. (2016). O papel da enfermagem no rompimento dos preconceitos LGBT nos serviços de saúde. In: $16^{\circ}$ congresso nacional de iniciação científica. http://conic-semesp.org.br/anais/files/2016/trabalho-1000022939.pdf

Brasil. Ministério da Saúde. Política nacional de saúde integral de lésbicas, gays, bissexuais, travestis e transexuais. $1^{\circ}$ Edição. Brasília, 2013.

Brasil. Ministério da Saúde. Mulheres lésbicas e bissexuais: Direitos, saúde e Participação Social. Brasília, 2013.

Brasil. Ministério da Saúde. Protocolos da Atenção Básica: Saúde das Mulheres. Brasília, 2016

Cabral, K. T. F., et al (2019). Assistência de enfermagem às mulheres lésbicas e bissexuais. Rev enferm UFPE on line., Recife, 13 (1) 9-85.

Crispim, J. E. B. (2018). Assistência de enfermagem à mulher lésbica e bissexual na atenção básica: protocolo de atendimento. Rev de pesquisa online Cuidado é fundamental Online. 10 34-39. Junho.

Eliason, M. J., et al (2013). Open the door for LGBTQ patients. Enfermagem 2019. 43 (8) 44-5.

Farias, G. M., et al (2018). Os cuidados do enfermeiro às lésbicas. Rev enferm UFPE on line. 12 (10) 2825-35, Recife.

Fernandes, M., Soler, L. D., Leite, M. C. B. P. (2018). Saúde das mulheres lésbicas e atenção à saúde: nem integralidade, nem equidade diante das invisibilidades. Rev. Bis. 19 (2). Dezembro.

Hadland, S. E, Yehia, B. R., Makadon, H. J. (2016). Caring for lgbtq youth in inclusive and affirmative environments. Pediatr Clin North Am. 63 (6) 955-969. Dezembro.

Jones-Schenk, J.; Bleich, M. R. (2018). Creating LGBTQ-Inclusive Care and Work Environments. The Journal of Continuing Education in Nursing. 49 (4).

Kano, M., et al (2016). Stakeholders' Recommendations to Improve Patient-centered "LGBTQ” Primary Care in Rural and Multicultural Practices. JABFM. 29 (1). Janeiro-Fevereiro.

Klotzbaugh, R.; Spancer, G, (2014). Magnet Nurse Administrator Attitudes and pportunities. JONA. 44 (9). Setembro.

Marshal, M. P. et al (2013). Mental Health and Substance Use Disparities Among Urban Adolescent Lesbian and Bisexual Girls. J Am Psychiatr Nurses Assoc. 19 (5) 271-279.

Matthews, A. K. et al (2013) The Relationships of Sexual Identity, Hazardous Drinking, and Drinking Expectancies with Risky Sexual Behaviors in a Community Sample of Lesbian and Bisexual Women. J Am Psychiatr Nurses Assoc. 19 (5) 259-270.

Mccann, E.; Sharek, D. (2014). Survey of lesbian, gay, bisexual, and transgender people's experiences of mental health services in Ireland. International Journal of Mental Health Nursing. 23 118-127.

Mendes, K. D. S.; Silveira, R. C. C. P.; Galvão, C. M. (2019). Uso de gerenciador de referências bibliográficas na seleção dos estudos primários em revisão integrativa. Texto \& Contexto Enfermagem. 28. 
Research, Society and Development, v. 10, n. 7, e6710716326, 2021

(CC BY 4.0) | ISSN 2525-3409 | DOI: http://dx.doi.org/10.33448/rsd-v10i7.16326

Moscheta, M. S.; Fébole, D. S.; Anzolin, B. (2016). Visibilidade seletiva: a influência da heterossexualidade compulsória nos cuidados em saúde de homens gays e mulheres lésbicas e bissexuais. Sau. \& Transf. Soc. 7 (3) 71-83, Florianópolis.

Oliveira, G. S., et al (2018). Acesso de lésbicas, gays, bissexuais e travestis/transexuais às Unidades Básicas de Saúde da Família. Rev Rene. 2018;

Richardson, B. P.; Ondracec, A. E.; Anderson, D. Do student nurses feel a lack of comfort in providing support for Lesbian, Gay, Bisexual or Questioning adolescents: what factors inflfluence their comfort level? Journal of Advanced Nursing, 73 (5) 1196-1207.

Silva, A. N.; Prata, M. (2018). O atendimento à mulher lésbica e a influência da heteronormatividade no cuidado.

Sousa, J. C., et al (2014). Promoção da saúde da mulher lésbica: cuidados de enfermagem. Rev Gaúcha Enferm. 35 (4) 108-13. Dez.

Zuzelo, P. R. (2014). Improving Nursing Care for Lesbian, Bisexual, and Transgender Women. JOGNN. (43) 520-530. 